\title{
Contabilidad creativa en Chile Una percepción de estudiantes y profesionistas ${ }^{1}$
}

\section{Fernando Morales}

\section{Parada}

Académico del Departamento de Administración y Auditoría, Facultad de Ciencias Empresariales, Universidad del Bio Bio.fmorales@ubiobio.cl.

\section{Reinier Hollander}

\section{Sanhueza}

Académico del Departamento de Administración y Auditoría, Facultad de Ciencias Empresariales, Universidad del Bio Bio.rholland@ubiobio.cl.

\footnotetext{
${ }^{1}$ Este artículo es resultado del proyecto de investigación 072416 4/R (2007-2008), el cual se desarrolló por las facilidades financieras y académicas que otorgó la Dirección de Investigación de la Universidad del Bío Bío.
}

\section{Resumen}

La contabilidad creativa ha resurgido como línea de investigación relevante en contabilidad, auditoría y ética de los negocios a partir de los escándalos financieros ocurridos en Estados Unidos, como Enron, Worldcom, Adelphia, Global Crossing, entre otros. El objetivo de este artículo, que forma parte de un proyecto de investigación más amplio realizado en Chile sobre el tema, pretende evaluar la percepción de profesionistas y estudiantes acerca de las posibilidades de la aplicación de contabilidad creativa en las normas y en la contabilidad de las empresas. Entre las conclusiones encontramos que los grupos de contadores y auditores externos poseen percepciones muy similares sobre la existencia y las posibilidades de manipular los estados financieros, pero también existen diferencias cuando las preguntas se orientan hacia la legitimidad de la contabilidad creativa, el aprovechamiento de ésta o si es común o no en Chile.

Palabras claves: contabilidad creativa, normas contables, Chile, auditoría. 


\title{
Creative accounting in Chile: a students and professionals perception
}

\begin{abstract}
Creative accounting has re-emerged as a relevant research subject in accounting, auditing, and business ethics. This is critical after the financial scandals, such as Enron, Worldcom, Adelphia, Global Crossing, in the United States. The present article is part of a major research project in Chile and its purpose is to evaluate the perception on the possibilities of applying creative accounting on national companies-standards and accounting. Among the conclusions we found that external accountants and auditors groups possess similar perceptions of the existence and possibilities of manipulating financial statements in Chile. However these groups responses differ when asking about the legitimacy of creative accounting, if they profit from it, and if it is common in Chile.
\end{abstract}

Keywords: creative accounting, accounting standards, Chile, auditing.

\section{Introducción}

Creemos necesario introducir brevemente el significado de la contabilidad como elemento base de la información empresarial y sobre la cual descansa la creatividad contable. Una visión general e internacional la proporciona el International Accounting Standard Board, IASB (1997), que en la Norma Internacional de Contabilidad $\mathrm{N}^{\mathrm{o}} 1$ declara que los estados financieros constituyen una representación estructurada de la situación financiera y del rendimiento financiero de la entidad, agregando que el objetivo de los estados financieros es suministrar información acerca de la situación financiera, del rendimiento financiero y de los flujos de efectivo de la entidad, que sea útil a una amplia variedad de usuarios a la hora de tomar decisiones económicas.

Laínez y Callao (1999), después de efectuar una revisión bibliográfica de definiciones sobre contabilidad creativa, conformaron una propia en la cual señalaron que la contabilidad creativa era la que aprovechaba las posibilidades que ofrecían las normas (opcionalidad, subjetividad y vacíos de regulación) para presentar estados financieros que reflejaran la imagen deseada y no necesariamente la que en realidad era. Posteriormente, hicieron una reflexión que resumía el conflicto, en ella afirmaron que la contabilidad creativa se encontraba entre el camino de las prácticas verdaderamente correctas y éticas y la legalidad o fraude, aunque aclaraban que era difícil delimitar dónde acababa la ética y dónde comenzaba la creati- 
vidad, así como dónde terminaba esta última y empezaba el fraude. Más adelante, Amat y Blake (1999) reconocieron este efecto como un proceso mediante el cual los contadores utilizaban el conocimiento de las normas para manipular las cifras incluidas en las cuentas de una empresa.

Otra aportación importante la efectuó Naser (1993), quien dijo que la contabilidad creativa era el proceso de manipular la contabilidad aprovechándose de las reglas y las opciones de valuación y revelación práctica para transformar los estados financieros de lo que eran a lo que debían ser. Además, aseguró que la flexibilidad en las valuaciones de contadores y normas de revelación siempre había permitido algún grado de creatividad. Para Jameson (1988) dicho concepto era esencialmente un proceso de usar las reglas, la flexibilidad proporcionada por ellas y las omisiones dentro de éstas. En este mismo sentido se encuentran los trabajos realizados por Healy y Wahlen (1999).

Por su parte, Vidal (2002) señaló que la contabilidad creativa correspondía a las actuaciones realizadas por la dirección de la empresa en el marco y total cumplimiento de los Principios de Contabilidad Generalmente Aceptados (PCGA), encaminadas a ofrecer la situación deseada en un momento determinado aprovechando la flexibilidad permitida en la normativa contable. A su vez, Cordobés y Molina (2000) presentaron una visión más moderada, afirmaron que si por creatividad se entendía la lectura del espíritu de la norma por encima de su letra, entonces consideraban que la contabilidad era creativa, de lo contrario los hechos descritos no reflejarían la sustancia económica que pretendían representar y la información sería menos útil para los stakeholders (Leuz et al., 2003).

Los alcances de la contabilidad creativa pueden darse en cualquier tipo de partida de los estados financieros, es decir, tanto en balance como en resultado (pérdidas y ganancias), además podrá afectar la valoración y/o la revelación donde existen múltiples posibilidades de llevarlas a cabo, como lo detallaron claramente Stlowy y Breton (2004). Por otra parte, la alteración contable puede implicar el mejoramiento o empeoramiento financiero (en cualquiera de las partidas) según sea el objetivo deseado de parte del manipulador, cuestión que se profundiza en el apartado siguiente. Es interesante resaltar que la contabilidad creativa se basa fundamentalmente en la aplicación ingeniosa de las normas y no en otras formas de manipulación para lograr estados financieros deseados por sobre lo que las normas en su aplicación adecuada resultarían. Algunos aspectos prácticos y legales en Estados Unidos se encuentran bien documentados en Mulford y Comiskey (2004) y Barreveld (2002). 


\section{Revisión de literatura}

Además de los trabajos señalados, se revisaron otros basados en encuestas a auditores con el objetivo de determinar el conocimiento y las posiciones de los auditores frente a este tema. Es así como Amat et al. (1997) presentaron algunas conclusiones desde una perspectiva española e inglesa ${ }^{2}$; por ejemplo, revelaron que $66 \%$ de los auditores españoles y $64 \%$ del Reino Unido consideraban a la contabilidad creativa como un problema importante; $85 \%$ de los encuestados del Reino Unido consideraban que este problema nunca sería solucionado, mientras que sólo $40 \%$ de los españoles compartían dicha visión. Pero aparecen diferencias entre los encuestados hispanos al considerarla como un problema en aumento: $37 \%$ reconoce que es cada vez más común en España frente a $42 \%$ que no la considera como un problema en aumento.

Por otra parte, Guevara y Cosenza (2004) también realizaron un trabajo de campo en el mismo sentido del anterior, pero con una muestra de auditores de diversos países ${ }^{3}$ aunque con algunas limitaciones por la alta heterogeneidad en las respuestas. De este trabajo destaca el alto grado de conocimiento sobre el concepto contabilidad creativa, pues $98 \%$ dijo conocerlo. Pero en contraste, a pesar de ser un asunto reconocido, $62.7 \%$ de los auditores no consideraban al momento de diseñar sus planes de auditoría la potencial existencia de prácticas creativas. A ello se suma $71.2 \%$ que afirmó no aplicar procedimientos de auditoría específicos para su detección, otro tanto señaló que no evaluaba, como parte del riesgo inherente, la existencia potencial de estas prácticas. En consecuencia, $61 \%$ de los entrevistados afirmó no tener algún protocolo establecido especialmente para cuando se detectaron estas prácticas. Más impresionante puede resultar que $42.4 \%$ de los encuestados aceptaron que los auditores participaban en el diseño de prácticas creativas a través de un departamento de consultoría siempre que este servicio fuera requerido por el cliente. Por tanto, de acuerdo con una legitimidad, que dejan ver los auditores encuestados por el estudio de Guevara y Cosenza, $64.41 \%$ cree que en sus informes de opinión sólo se debe hacer mención de las prácticas creativas cuando éstas incumplan los PCGA y nadie $(0 \%)$ considera incluirla siempre y sin condición.

\footnotetext{
${ }^{2}$ Este trabajo se desarrolló en España, para ello se enviaron cien cuestionarios a auditores, de los cuales sólo se obtuvieron 29 respuestas. La parte de Reino Unido se obtuvo de dos trabajos realizados por Naser (1993), quien en un primer estudio reclutó 22 respuestas y en un segundo otras 20. La base del Reino Unido, entonces, se obtiene considerando las 42 respuestas.

${ }^{3}$ Este trabajo obtuvo las siguientes respuestas: Argentina 7, Brasil 18, Colombia 9, Estados Unidos 4 y Venezuela 21. El objetivo inicial era obtener al menos 200 respuestas, pero sólo se lograron 59.
} 
En Argentina ${ }^{4}$, Huber (1999) descubrió que 54\% de sus entrevistados consideraba el uso de la contabilidad creativa como una herramienta legítima y muy conocida en ese país; $41 \%$ de los encuestados se inclinó en descartar la existencia de estas prácticas al elaborar estados contables para entidades financieras y 35\% cuando estos estados contables tienen fines fiscales. Finalmente, consideramos interesante incluir la respuesta de la siguiente pregunta: ¿Considera usted que la diversidad de párrafos de dictamen [...] libera la responsabilidad del auditor frente a las prácticas de contabilidad creativa?, donde $73 \%$ respondió de forma afirmativa y el restante $23 \%$ contestó que no. Podemos afirmar que además del problema con la subjetividad del tema, el trabajo de auditoría y quizá el propio informe final, donde emite la opinión el auditor, podrían tener sesgos relacionados con la forma de enfrentar esta problemática.

Para culminar esta breve revisión bibliográfica presentamos investigaciones sobre la opinión de auditores y contadores acerca de la legitimidad que tienen las prácticas de contabilidad creativa. En España, Amat y Blake (1999) estudiaron nueve encuestas de un total de 29 auditores que reconocían la contabilidad creativa como prácticas lícitas (31\%). Para el caso inglés, Naser (1993) encontró una aceptación de 46\%, mientras que Fortes (Guevara y Consenza, 2004: 19) al encuestar a los auditores en Portugal encontró que $24 \%$ legitimaban estas prácticas. Otro estudio, que también consideró a un país europeo, lo efectuó Baralexis (2004), quien tras encuestar a profesionistas de la auditoría y contabilidad en Grecia, obtuvo 76\% y $71 \%$ de aceptación como lícitas a las prácticas creativas.

Para el caso de América, consideramos el estudio realizado por Guevara y Cosenza (2004) donde 32 de 59 auditores de distintos países de América consideraban a la contabilidad creativa como una estrategia contable de la organización, y por ende, estos autores consideraban que existía una legitimación del asunto. Huber descubrió para el caso de Argentina que 54\% de sus encuestados legitimaban las prácticas de contabilidad creativa.

En las investigaciones señaladas no se observa una respuesta predominante, pues de los cuatro países europeos sólo Grecia destaca con $76 \%$ porque sus encuestados consideraban a la contabilidad creativa como una práctica legitima; en cambio,

\footnotetext{
${ }^{4}$ El cuestionario se dirigió a 20 despachos de auditores de Argentina, obteniendo respuesta de doce. La selección de los despachos se realizó con el criterio del Consejo Profesional de Ciencias Económicas de Santa Fe (CPCESF)
} 
los otros países muestran un bajo porcentaje: Reino Unido $46 \%{ }^{5}$, España $31 \%$ y Portugal 24\%. Por otro lado, los países latinoamericanos contestaron que las prácticas creativas son legítimas (54\%), Estados Unidos es incluido en el estudio de Guevara y Cosenza con sólo cuatro encuestados.

\section{Metodología}

La presente investigación pretende conocer la percepción que tienen los profesionistas y estudiantes de la licenciatura en contabilidad sobre la existencia de las prácticas de contabilidad creativa que a priori es posible que ocurra en Chile, según la revisión bibliográfica efectuada.

Basándonos en las evidencias obtenidas por otros autores y en la existencia de posibilidades de aplicación creativa en la norma chilena, formulamos las siguientes hipótesis de trabajo:

$H_{O I}$ : Los auditores tienen igual percepción que los contadores sobre las posibilidades de usar la contabilidad creativa en Chile.

$H_{02}$ : Los auditores tienden a legitimar en igual medida que los contadores las prácticas de contabilidad creativa en Chile.

$H_{03}$ : El reconocimiento de prácticas contables creativas y legitimidad de la misma es igual entre todos los profesionistas y estudiantes.

$H_{04}$ : El reconocimiento de prácticas contables creativas y legitimidad de la misma es igual entre todos los encuestados, sin importar sus experiencias laborales.

Se aplicó una encuesta, seleccionada por conveniencia, a través de un cuestionario a una muestra de profesionistas y estudiantes. El instrumento que se preparó exclusivamente para esta investigación se revisó con anterioridad, además, se tomó como base una parte del trabajo de Naser (1993). El cuestionario (ver Anexo) que se aplicó consistía de13 preguntas que sólo permitían respuestas cerradas según la escala Liker ${ }^{6}$ de cinco puntos. El procesamiento de las encuestas se realizó mediante el software SPSS 12.0, donde se realizaron cuadros de frecuencia, cuadros de contingencia (utilizando el estadístico $X_{2}$ de Pearson), análisis cluster y factorial.

\footnotetext{
${ }^{5}$ Puede consultarse más sobre el caso de UK en Shah, 1996;1998.

${ }^{6}$ Escala utilizada: (1) Totalmente en desacuerdo; (2) Desacuerdo; (3) No opinó; (4) Acuerdo; (5) Totalmente de acuerdo, y una escala reducida de 1 a 3 para otras preguntas: (1) Nada; (2) Algo; (3) Mucho.
} 
En el instrumento utilizado se consideraron las preguntas 1 a 8 relacionadas con el conocimiento sobre la contabilidad creativa, que permitían contrastar $H_{0 I}$. Por otra parte, las preguntas 9 a 13 se relacionaban con la legitimación de la contabilidad creativa y, en consecuencia, con la $H_{02}$. Para el contraste de las restantes hipótesis se utilizaron las 13 primeras preguntas del cuestionario.

La muestra de profesionistas y estudiantes por encuestar se obtuvo en colaboración con la Universidad del Bío Bío (Chile). El universo de profesionistas por encuestar fue de 151 (de un total de aproximadamente 550 egresados) a quienes se les realizaron tres envíos ${ }^{7}$ del cuestionario. Finalmente, se obtuvieron 53 respuestas, es decir, $35 \%$ del total de profesionistas a quienes tuvimos posibilidad de encuestar. Con relación a los estudiantes, sólo se aplicó el cuestionario a un grupo en aula ${ }^{8}$, de ahí se obtuvieron 38 cuestionarios.

\section{Resultados}

Antes de profundizar en los resultados, presentamos las características de los profesionistas encuestados (53 en total), clasificados por actividad profesional, experiencia laboral total y actividad actual, lo que se presenta en el cuadro 1.

\section{Cuadro 1}

\section{Característica de los profesionistas encuestados}

\begin{tabular}{ccccc|cccc}
\hline & \multicolumn{4}{c}{ Experiencia total } & \multicolumn{4}{c}{ Experiencia en la actividad actual } \\
& $\begin{array}{c}\text { Menos } \\
\text { de 3 años }\end{array}$ & $\begin{array}{c}\text { Entre 3 y 5 } \\
\text { años }\end{array}$ & $\begin{array}{c}\text { Mas de } \\
\mathbf{5} \text { años }\end{array}$ & Total & $\begin{array}{c}\text { Menos de } \\
\text { 3 años }\end{array}$ & $\begin{array}{c}\text { Entre 3 y } \\
\mathbf{5} \text { años }\end{array}$ & $\begin{array}{c}\text { Mas de } \\
\mathbf{5} \text { años }\end{array}$ & Total \\
\hline Auditor externo & 2 & 1 & 5 & $\mathbf{8}$ & 5 & 1 & 3 & 9 \\
$\begin{array}{c}\text { Auditor interno } \\
\text { Contadores }\end{array}$ & 1 & 1 & 4 & $\mathbf{6}$ & 5 & 0 & 1 & 6 \\
$\begin{array}{c}\text { Otras activ. } \\
\text { profesionales }\end{array}$ & 3 & 5 & 9 & $\mathbf{1 7}$ & 12 & 4 & 3 & 19 \\
\hline $\begin{array}{c}\text { Sub total } \\
\text { Perdidos }\end{array}$ & $\mathbf{8}$ & $\mathbf{1 3}$ & $\mathbf{2 7}$ & $\mathbf{4 8}$ & $\mathbf{3 2}$ & 7 & $\mathbf{1 2}$ & $\mathbf{5 1}$ \\
Total & $\mathbf{8}$ & $\mathbf{1 3}$ & $\mathbf{2 7}$ & $\mathbf{5 3}$ & $\mathbf{3 2}$ & 7 & $\mathbf{1 2}$ & $\mathbf{5 3}$ \\
\hline
\end{tabular}

\footnotetext{
${ }^{7}$ Vía correos electrónicos proporcionados por el Director de Carrera. Los envíos fueron: primero 09/03/2006, segundo 16/03/2006 y tercer envío 03/04/2006.

${ }^{8}$ Los estudiantes se seleccionaron de un curso de $5^{\circ}$ semestre, quienes ya habían cursado contabilidad, pero no los cursos de auditoría de cuentas (o de estados financieros). Estos alumnos sólo representan aproximadamente el 12,66\% el total de estudiantes de la carrera de Contador Público y Auditor de la Universidad del Bío Bío, sede Concepción.
} 
Considerando que la actividad profesional de "auditor" se dividió en externo (de estados financieros) e interno, se puede observar un equilibrio entre tres grupos de encuestados, donde a nivel de experiencia total como los auditores son $29 \%$ y los contadores de empresas y quienes desarrollan otras actividades profesionales $35 \%$, respectivamente. La mayoría de los profesionales encuestados poseen una experiencia total superior a cinco años, lo que nos otorga una mayor confianza en cuanto a la calificación del encuestado.

La segunda parte del cuestionario, preguntas 14 a 18, permitió formarnos una idea general de la percepción de los encuestados sobre la contabilidad creativa. En las respuestas se observó que de existir manipulación de estados financieros, los encuestados se inclinarían porque habría "mucha" ocurrencia en las pequeñas empresas (47.1\%), "algo" (59.8\%) en empresas cotizadas, mientras que sobre las empresas no cotizadas se encontró una división en las respuestas entre "mucho" (42.5\%) y "algo" (47.1\%).

En este sentido, observamos cómo los encuestados consideraron "mucha" responsabilidad en la manipulación contable al "gobierno y administración de la empresa gerencia y/o directorio" (64.4\%), mientras que a los reguladores en torno a la contabilidad, como el Colegio de Contadores de Chile A.G., creen que no tienen "nada" de responsabilidad (41.4\%).

Asimismo, quisimos indagar sobre las partidas contables que serían más proclives a la manipulación, presentándoles una relación de ítems de las cuentas de activo, pasivo y resultados para buscar en sus respuestas alguna mayor o menor identificación en las prácticas de manipulación. Salvo la "depreciación de activos" que obtuvo iguales porcentajes en "algo" y "mucho" (36\%), "reconocimiento de ingresos" que tuvo mayor inclinación con $41 \%$ y "transacciones con empresas vinculadas" que lo consideraban como "mucho" en la realización de estas prácticas, el resto de ítems se concentraron en la opción de respuesta central "algo", lo que nos limita con claridad la percepción de los encuestados.

Otro asunto interesante que percibimos en las respuestas es la percepción que tienen los encuestados sobre la expectativa de la próxima incorporación de las Normas Internacionales de Información Financiera (NIIF); la mayoría consideraba que con estas normas ocurrirían cambios a favor de limitar la contabilidad creativa. Es así como perciben que la opcionalidad, subjetividad y vacíos normativos se limitarán "mucho" $31 \%, 35.6$ y $32.2 \%$, respectivamente; mientras que por "algo" se inclinaron $54 \%, 49.4 \%$ y $52.9 \%$. 
Después de realizar cuadros de contingencia por actividad en las preguntas 1 a 9 , destacamos algunos resultados que contribuirán a profundizar en la percepción de los encuestados sobre el asunto de esta investigación. Es así como observamos en las preguntas 1 y 2 , analizadas en conjunto por estar referidas a un mismo asunto, que los grupos de auditores externos y contadores contestaron de forma similar y aceptada (70\%).

Con respecto a las preguntas 4 y 5 , ambas referidas a las posibles subjetividades existentes en la normativa, nuevamente existió cierta coincidencia de respuesta entre los auditores externos y los contadores; los auditores son los más contundentes en sus respuestas afirmativas, superando en aproximadamente un $10 \%$ a los contadores.

Sin embargo, cuando nos referimos a la posible existencia de vacíos normativos, lo cual podría ser una herramienta de manipulación, $100 \%$ de los auditores externos se mostraron de acuerdo, además, $89 \%$ consideraron que ante un vacío normativo es adecuado recurrir a las normas internacionales. Por otro lado, $61 \%$ de los contadores consideraron que dichos vacíos permiten la manipulación y $78 \%$ señalaron que se debían recurrir a normas internacionales.

Al preguntar si los contadores pueden aprovecharse de estas características de la contabilidad (opcionalidad, subjetividad y vacíos normativos) por estar dentro del marco normativo, los auditores externos contestaron contundentemente "total desacuerdo" (89\%), mientras que los contadores no se mostraron muy claros y su respuesta se distribuyó entre (39\%) "desacuerdo" y "total desacuerdo; 33\% no opinó y sólo $28 \%$ se inclinó por el total acuerdo. Algo similar ocurrió al preguntar si en Chile es común la manipulación contable; los auditores contestaron en desacuerdo (56\%), mientras que los contadores están de acuerdo (39\%). Así también con respecto al reconocimiento de la contabilidad creativa como legítima herramienta de negocio, $67 \%$ de los auditores se mostraron "totalmente en desacuerdo", mientras que los contadores se dividieron: $33 \%$ de acuerdo y $33 \%$ en desacuerdo.

En relación con el total de respuestas (profesionistas 60.92\% y estudiantes 39.08\%), se presentan en el cuadro 2 las estadísticas descriptivas para tener una idea general de las respuestas obtenidas. 
Cuadro 2

Estadísticas de las respuestas

\begin{tabular}{cccccccc}
\hline $\begin{array}{c}\text { Preguntas/ } \\
\text { Variables }\end{array}$ & $\begin{array}{c}\text { Datos } \\
\text { Válidos }\end{array}$ & $\begin{array}{c}\text { Datos } \\
\text { Perdidos }\end{array}$ & Media & Desv. típ. & Varianza & Mínimo & Máximo \\
\hline P01 & 87 & 0 & $\mathbf{3 , 6 2 1}$ & 1,059 & 1,122 & 1 & 5 \\
P02 & 85 & 2 & $\mathbf{3 , 6 7 1}$ & 1,005 & 1,009 & 1 & 5 \\
P03 & 86 & 1 & $\mathbf{3 , 1 8 6}$ & 1,101 & 1,212 & 1 & 5 \\
P04 & 85 & 2 & $\mathbf{3 , 8 3 5}$ & 1,010 & 1,020 & 1 & 5 \\
P05 & 86 & 1 & $\mathbf{4 , 1 5 1}$ & 0,940 & 0,883 & 1 & 5 \\
P06 & 85 & 2 & $\mathbf{3 , 6 3 5}$ & 0,962 & 0,925 & 1 & 5 \\
P07 & 86 & 1 & $\mathbf{4 , 0 7 0}$ & 0,968 & 0,936 & 1 & 5 \\
P08 & 85 & 2 & $\mathbf{3 , 1 4 1}$ & 1,255 & 1,575 & 1 & 5 \\
P09 & 85 & 2 & $\mathbf{3 , 8 3 5}$ & 1,045 & 1,092 & 1 & 5 \\
P10 & 86 & 1 & $\mathbf{2 , 8 1 4}$ & 1,279 & 1,636 & 1 & 5 \\
P11 & 85 & 2 & $\mathbf{3 , 4 1 2}$ & 1,105 & 1,221 & 1 & 5 \\
P12 & 86 & 1 & $\mathbf{3 , 0 4 7}$ & 1,217 & 1,480 & 1 & 5 \\
P13 & 86 & 1 & $\mathbf{3 , 5 4 7}$ & 1,165 & 1,357 & 1 & 5 \\
\hline
\end{tabular}

En el cuadro 3, la pregunta 4 está relacionada con la subjetividad que se puede encontrar en los BT. Se puede afirmar que los encuestados percibieron una mayor subjetividad en las normas por sobre las opcionalidades; la pregunta 3, relacionada con las opciones permitidas en la norma, tuvo un nivel de respuesta con menor "acuerdo" que la pregunta 4.

En el caso de la pregunta 5, relacionada con el uso del criterio conservador ante subjetividades y controversias en las normas, la tendencia a contestar de acuerdo se puede entender como un cumplimiento del primer PCGA en la norma chilena es "equidad" ; además, el conservadurismo de criterio se precisa en otro principio, "criterio prudencial"10. En conclusión, existe relación entre la mayor frecuencia de contestación de acuerdo con la realidad normativa al respecto.

En cuanto a la número 7, que pregunta si en un caso de vacío normativo es adecuado recurrir a las normas internacionales, el mayor número de respuestas fue "acuerdo" y "muy de acuerdo". Esto se debe a que desde 1997 está vigente en Chile el вт N56 que establece el uso de las NIC ante vacíos normativos en los BT.

\footnotetext{
9 "Los estados financieros deben prepararse de tal modo que reflejen con equidad los distintos intereses en juego en una entidad dada. Este principio en el fondo es el postulado básico o principio fundamental al que está subordinado el resto [...]".

10 "La preparación de estados financieros, por lo tanto, requiere que un criterio sano sea aplicado en la selección de la base a emplear para lograr una decisión prudente. Esto involucra que ante dos o más
} 


\section{Cuadro 3}

Frecuencias de respuestas con mayores medias

\begin{tabular}{ccccccc|cccccc}
\hline & P04 & & & & & & P05 & & & \\
& 1 & 2 & 3 & 4 & 5 & Total & 1 & 2 & 3 & 4 & 5 & Total \\
\hline Auditor externo & 1 & 0 & 0 & 5 & 3 & $\mathbf{9}$ & 0 & 0 & 0 & 4 & 5 & $\mathbf{9}$ \\
Auditor interno & 0 & 0 & 0 & 4 & 2 & $\mathbf{6}$ & 0 & 0 & 0 & 0 & 6 & $\mathbf{6}$ \\
Contable & 0 & 3 & 1 & 10 & 4 & $\mathbf{1 8}$ & 2 & 0 & 0 & 13 & 3 & $\mathbf{1 8}$ \\
Otras actividades profesionales & 0 & 1 & 0 & 12 & 3 & $\mathbf{1 6}$ & 0 & 2 & 1 & 10 & 4 & $\mathbf{1 7}$ \\
Sin trabajo actual & 0 & 0 & 0 & 1 & 1 & $\mathbf{2}$ & 0 & 0 & 0 & 1 & 1 & $\mathbf{2}$ \\
Estudiante & 1 & 8 & 2 & 17 & 6 & $\mathbf{3 4}$ & 1 & 1 & 4 & 14 & 14 & $\mathbf{3 4}$ \\
Total & 2 & 12 & 3 & 49 & 19 & $\mathbf{8 5}$ & 3 & 3 & 5 & 42 & 33 & $\mathbf{8 6}$ \\
\hline & $\mathbf{P 0 7}$ & & & & & & P09 & & & & \\
Auditor externo & 1 & 2 & 3 & 4 & 5 & Total & 1 & 2 & 3 & 4 & 5 & Total \\
Auditor interno & 0 & 1 & 0 & 3 & 5 & $\mathbf{9}$ & 0 & 0 & 0 & 5 & 4 & $\mathbf{9}$ \\
Contable & 0 & 0 & 1 & 2 & 3 & $\mathbf{6}$ & 0 & 1 & 1 & 1 & 3 & $\mathbf{6}$ \\
Otras actividades profesionales & 1 & 0 & 3 & 10 & 4 & $\mathbf{1 8}$ & 0 & 3 & 3 & 8 & 4 & $\mathbf{1 8}$ \\
Sin trabajo actual & 0 & 2 & 2 & 9 & 4 & $\mathbf{1 7}$ & 1 & 4 & 3 & 5 & 4 & $\mathbf{1 7}$ \\
Estudiante & 0 & 0 & 0 & 1 & 1 & $\mathbf{2}$ & 0 & 0 & 0 & 2 & 0 & $\mathbf{2}$ \\
Total & 1 & 2 & 4 & 12 & 15 & $\mathbf{3 4}$ & 0 & 3 & 8 & 11 & 11 & $\mathbf{3 3}$ \\
\hline & 2 & 5 & 10 & 37 & 32 & $\mathbf{8 6}$ & 1 & 11 & 15 & 32 & 26 & $\mathbf{8 5}$ \\
\hline
\end{tabular}

Nota: 1: Totalmente en desacuerdo; 2: Desacuerdo; 3: No opino; 4: Acuerdo; 5: Totalmente de acuerdo

En la pregunta 9 los encuestados contestaron frecuentemente "acuerdo", y los auditores externos "totalmente de acuerdo" en que no deben permitir que sus clientes se aprovechen de las posibles opcionalidades, subjetividades y vacíos normativos, lo cual dista de la responsabilidad de estos profesionistas en la contabilidad creativa. Anteriormente, señalamos que sólo un tercio de los encuestados afirmó que son muy responsables y el resto lo consideró algo o nada responsable.

En contraste a las preguntas que obtuvieron sobre promedio y que analizamos en los párrafos anteriores, se encuentra la pregunta 10 con un bajo promedio que destaca sobre los demás. Esta pregunta indaga acerca de la percepción de los encuestados sobre la validez que tiene para los contadores efectuar alteraciones contables dentro del marco normativo, a lo que los encuestados tendieron a estar en "desacuerdo", en especial los auditores externos y hubo menor énfasis entre los contadores y estudiantes. 


\section{Contrastes para hipótesis $H_{01}$ y $H_{02}$}

Con respecto a las hipótesis planteadas para este trabajo, recordamos que $H_{01}$ y $H_{02}$ son similares entre sí, pues buscan reconocer similitudes o diferencias entre el grupo de contadores versus auditores encuestados; en consecuencia, para las hipótesis se consideraron las preguntas relacionadas al conocimiento sobre contabilidad creativa de P01 hasta P08, que servirán para contrastar $H_{0 I}$ y las preguntas P09 hasta P13 relacionadas a percepción sobre legitimidad de la contabilidad creativa que servirán para contrastar $H_{02}$. Los resultados de aplicar el estadístico de $X_{2}$ de Pearson para esos grupos se presentan en el cuadro 4.

\section{Cuadro 4}

Chi-cuadrado de Pearson para contraste de $\boldsymbol{H}_{01}$ y $\boldsymbol{H}_{02}$

\begin{tabular}{|c|c|c|c|c|c|c|c|c|c|c|c|c|c|}
\hline & P01 & $\mathrm{P} 02$ & $\mathrm{P} 03$ & P04 & P05 & P06 & $\mathrm{P} 07$ & P08 & P09 & P10 & P11 & P12 & P13 \\
\hline $\mathrm{N}$ & 34 & 33 & 33 & 33 & 33 & 33 & 33 & 33 & 33 & 33 & 33 & 33 & 33 \\
\hline P-valor & .138 & .747 & .167 & .294 & .004 & .152 & .217 & .932 & .415 & .257 & .059 & .081 & .052 \\
\hline
\end{tabular}

De acuerdo con los resultados, y basándonos en el P-valor de las variables (preguntas) P01 a P08, observamos que en su mayoría son muy altos, lo que no nos permite rechazar la hipótesis nula $H_{01}$, a un nivel de confianza del $5 \%$. Por lo tanto, salvo P05 con un 0.004 que indica que habría evidencia para validar la existencia de diferencias significativas entre los grupos y, en consecuencia, sólo para esta variable rechazamos la hipótesis nula. El resto de variables suponen la no existencia de diferencias significativas que nos impulsan a aceptar la hipótesis, que los auditores tienen la misma percepción que los contadores sobre las posibilidades de contabilidad creativa en Chile.

Con respecto de P09 a P13, que sirven para contrastar $H_{02}$, no podemos rechazar la hipótesis nula planteada, pues no existen diferencias significativas en la totalidad de las variables. Por lo tanto, de acuerdo con los resultados, y considerando un nivel de confianza del 5\%, los auditores encuestados tienden a legitimar en igual medida que los contadores las prácticas de contabilidad creativa en Chile.

\section{Contrastes para hipótesis $H_{03}$}

Sobre $H_{03}$ nuestro objetivo es evidenciar posibles diferencias de percepción sobre el asunto entre el grupo de profesionistas encuestados, independientemente de su situación laboral, versus el grupo de estudiantes de contabilidad que a priori com- 
prenderían menos del asunto. Para este caso, se consideran las 13 preguntas del cuestionario, los resultados se presentan en el cuadro 5.

\section{Cuadro 5}

\section{Chi-cuadrado de Pearson para contraste de $\boldsymbol{H}_{03}$}

\begin{tabular}{cccccccccccccc}
\hline & P01 & P02 & P03 & P04 & P05 & P06 & P07 & P08 & P09 & P10 & P11 & P12 & P13 \\
\hline $\mathrm{N}$ & 87 & 85 & 86 & 85 & 86 & 85 & 86 & 85 & 85 & 86 & 85 & 86 & 86 \\
P-valor & .254 & .026 & .048 & .226 & .365 & .112 & .810 & .356 & .561 & .168 & .518 & .263 & .173 \\
\hline
\end{tabular}

Prácticamente, todas las variables indican que no podemos rechazar la hipótesis nula $H_{03}$, con excepción de P02 y P03 que supondrían un rechazo y, en consecuencia, en esas variables en particular existirían diferencias significativas entre los dos grupos analizados. En conclusión, para esta hipótesis no existirían diferencias significativas que permitieran rechazar la formulación de igualdad entre los grupos; en cuanto al reconocimiento de prácticas contables creativas y legitimidad de la misma es igual entre todos los profesionistas encuestados y el grupo de estudiantes.

\section{Contrastes para hipótesis $H_{04}$}

Sobre $H_{04}$, nuestra intención es evaluar todo el grupo de profesionistas (independiente de su actividad) basándonos en su experiencia laboral, donde a priori podrían existir diferencias entre aquellos con mayor experiencia con relación a los que poseen menos. Se aplicó la prueba de chi-cuadrado, para 13 variables, misma que se presenta en el cuadro 6.

\section{Cuadro 6}

Chi-cuadrado de Pearson para contraste de $\boldsymbol{H}_{04}$

\begin{tabular}{cccccccccccccc}
\hline & P01 & P02 & P03 & P04 & P05 & P06 & P07 & P08 & P09 & P10 & P11 & P12 & P13 \\
\hline $\mathrm{N}$ & 48 & 47 & 47 & 46 & 47 & 47 & 47 & 46 & 47 & 47 & 46 & 47 & 47 \\
P-valor & .000 & .009 & .494 & .175 & .133 & .012 & .179 & .644 & .517 & .992 & .649 & .232 & .716 \\
\hline
\end{tabular}

Los resultados no distan mucho de lo observado en las anteriores validaciones, pues sólo existen tres P-valores significativos que permitan rechazar la hipótesis nula en P01, P02 y P06. Por lo tanto, de acuerdo con la observación del resto de variables, que son mayoritarias, no podemos rechazar $H_{04}$, lo que significa que habría igualdad en el reconocimiento de prácticas contables creativas y legitimidad de la misma, entre todos los encuestados, sin importar sus experiencias laborales 


\section{Conclusiones}

Este trabajo se basó en la búsqueda de evidencia empírica sobre la percepción de la contabilidad creativa por diferentes grupos de encuestados que tiene vinculación con la disciplina contable desde distintas perspectivas. Asimismo, pretendíamos comparar si la percepción de la muestra de profesionistas y estudiantes de contabilidad sobre la existencia de prácticas de manipulación en normas chilenas tenían similitudes o diferencias significativas bajo diferentes agrupaciones. Trabajos previos de este tipo se han realizado con muestras de profesionistas principalmente en Estados Unidos, países europeos y algunos países latinoamericanos, pero no se han encontrado trabajos aplicados al caso chileno en particular.

Con base en las respuestas obtenidas de los cuestionarios y tras realizar un análisis estadístico de las mismas, observamos que efectivamente los encuestados, en general, reconocen la existencia de estas posibilidades en tres niveles: opcionalidad, subjetividad y vacíos normativos, aunque el primero de ellos de forma más leve que los otros dos. De igual forma, hemos observado que los grupos de contadores y auditores externos, ambos altamente sensibles en temas manipulación contable por su vinculación directa en la elaboración de información financiera o como revisores de la misma, poseen percepciones muy similares sobre la existencia y las posibilidades de manipular los estados financieros en Chile, pero esta igualdad en las respuestas se distancian cuando las preguntas se orientan hacia la legitimidad de la contabilidad creativa, el aprovechamiento de ésta o si es común o no en Chile, en cuyo caso los auditores externos tienden a estar en desacuerdo con dichas afirmaciones, mientras que los contadores tienden a aceptarlo, aunque no con tanta fuerza.

En general, se observó que no existen diferencias significativas entre las percepciones de los grupos de auditores y contadores al procesar la totalidad de las preguntas; además, analizamos los grupos de profesionistas versus estudiantes y profesionistas con mayor y menor experiencia; en general, no rechazamos las hipótesis de existencia de diferencias entre los grupos, por lo que descubrimos que sí había variables donde los grupos son significativamente diferente entre ellos.

Las escasas diferencias descubiertas al comparar las respuestas de los grupos sugiere que el conocimiento y/o percepción de la contabilidad creativa estaría por sobre la pericia y experiencia profesional y es reconocida por todos sin importar su actividad profesional ni años de experiencia, aun los estudiantes igualan sus 
respuestas con los profesionistas. También habría que considerar que dos muestras (estudiantes y profesionistas) son de una misma nacionalidad y, por ende, se encuentran inmersos en la misma cultura, estas percepciones ya habían sido descubiertas por Geiger et al. (2007), las cuales se podrían aplicar al caso de Estados Unidos y México.

Un aspecto interesante de los resultados del análisis factorial es que el factor que agrupa las variables sobre legitimidad y ética en las prácticas creativas pierde fuerza con relación a las variables que tratan los aspectos propiamente normativos. Podemos concluir que a pesar de ser evidente la existencia de contabilidad creativa en la norma chilena, y al ser perfectamente reconocida por los encuestados no es muy aceptada, y el debate existente en la literatura internacional sobre el correcto o incorrecto uso de estas prácticas también se da en Chile.

Sin embargo, se deben circunscribir estas conclusiones al carácter localista del estudio con una muestra que no permite inferir sus resultados a la población y que, por tanto, debe considerase un primer paso para ampliar el objetivo general del estudio a nuevas investigaciones.

De esta forma, creemos interesante proponer para futuros trabajos de investigación la cercana e importante relación de la contabilidad creativa con otras líneas de investigación, donde algunos puntos se entrecruzan y podrían generar cuerpos de conocimientos muy interesantes; por ejemplo, aspectos relativos al rol de los reguladores contables, mitos en la enseñanza de las materias contables (considerando que exista un aspecto intuitivo de parte del estudiante acerca de la contabilidad) y los siempre delicados aspectos éticos, los cuales ya han sido investigados en trabajos de Gowthorpe y Amat (2005), Amat et al. (1999) y Blake et al. (2002).

\section{Referencias}

Amat, o., J. Blake y J. Dowds (1999). "The ethics of creative accountings". Econommics workings papers. http://www.econ.upf.edu/docs/papers/downloads/349.pd fon-line [08.12.2008]. 2000. 
79:24-31.

BARAleXIS, S. (2004). "Creative accounting in small advancing countries: The Greek case". Managerial Auditing Journal, 19-3:440-461.

BARreveld, D. (2002). The Enron collapse: Creative accounting, wrong economics or criminal acts?, Estados Unidos: Universe.

Blake, J., R. Bond y E. Oliveras (2002). "Ethics of creative accounting - Some Spanish evidence". Business Ethics, Vol. 9-3:136-142.

Cordobés, M. y H. Molina (2000). "Algunas reflexiones sobre la contabilidad creativa". Revista Técnica Contable, febrero, 89-110.

Geiger, M., C. Quirvan y A. Hazera (2007). "An international comparison of student perceptions of earnings management". Contaduría y Administración, No. 223:53-68.

Guevara, I. y J. Consenza (2004). "Los auditores independientes y la contabilidad creativa: Estudio empírico comparativo". Revista Compendium, julio, $5-24$.

Growthorpe, C. y O. Amat (2005). "Creative accounting: Some ethical issues of macro and Micro manipulations". Journal of Business Ethics, Vol. 57$1: 55-64$.

Huber, G. (1999). Contabilidad creativa. Una aproximación empírica. Argentina: Centro de Publicaciones de la Universidad Nacional del Litoral.

INTERNATIONAL ACCOUNTING STANDARD BOARD (1997). Presentación de Estados Financieros $N^{\circ} 1$. Londres: Norma Internacional de Contabilidad.

JAMESON, M. (1988). A practical guide to creative accounting. Reino Unido: Kogan Page.

HeAly, P. y J. WAHLEN (1999). "A review of the earnings management literature and its implications for standard setting". Accounting Horizons, Vol.134:365-383. 
Laínez, J. y S. Callao (1999). Contabilidad creativa. España: Civitas.

Leuz, C., D. NANDAB y P. Wysocki (2003). "Earnings management and investor protection: an international comparison". Journal of Financial Economics, Vol. 69-3:505-527.

Mulford, Ch. y E. Comiskey (2002). The financial numbers game, Estados Unidos: Wiley.

NASER, K. (1993). Creative financial accounting: Its nature and use, Reino Unido: Prentice Hall.

SHAH, A. (1998). "Exploring the influences and constraints on creative accounting in the United Kingdom". European Accounting Review, Vol. 7-1:83-104.

(1996). "Creative compliance in financial reporting". Accounting, Organizations and Society, Vol.21-1:23-39.

Stlowy, H. y G. Breton (2004). "Accounts manipulation: A literature review and proposed conceptual framework". Review of Accounting \& Finance, Vol.3-1:5-66.

VIDAL, M. (2002). "Reflexiones en torno a la contabilidad creativa". Revista Técnica Contable, 54-647:833-845. 


\section{ANEXO}

\section{Cuestionario aplicado}

\section{1-Muy en desacuerdo 2-Desacuerdo 3-No opino 4-De acuerdo 5-Muy de acuerdo}

1. En las normas contables, contenida en los вт del Colegio de Contadores, existen distintas alternativas (opcionalidades) al momento de registrar un hecho económico.

2. Existen BT que dan mayores posibilidades de alternativas (opcionalidades) al momento de registrar hechos económicos.

3. Ante una situación de opcionalidad permitida por un вт, se puede alternar procedimientos contables a través de los años basados en las propias opciones que otorga un BT.

4. Es frecuente encontrar en los BT situaciones que denotan cierta subjetividad (incertidumbres de cómo aplicar lo que la propia normativa indica) al momento de efectuar contabilizaciones.

5. Ante situaciones de subjetividad y controversia en las propias normas, el contador y/o auditor debe optar por la más conservadora (en términos de PCGA).

6. En las normas contables, contenidas en los BT, existen vacíos normativos, es decir, temas o hechos económicos que no tratan cómo valorizarse y/o presentarse.

7. Ante un vacío normativo en los BT, es adecuado recurrir a las Normas Internacionales de Contabilidad en búsqueda de una norma, o bien, a una norma reconocida por agrupaciones profesionales en otros países.

8. Ante un vacío normativo en los BT, es adecuado aplicar el criterio profesional para contabilizar la situación no normada.

9. Efectuar alteraciones contables "dentro de las propias normas", aprovechándose de posible opcionalidades, subjetividades y vacíos, es algo que los auditores externos no deben permitir en sus clientes.

10. Efectuar alteraciones contables "dentro de las propias normas", aprovechándose de posibles opcionalidades, subjetividades y vacíos, es algo que Contadores pueden efectuar por estar dentro del marco regulador.

11. Es común en Chile, la manipulación de estados financieros dentro del propio marco normativo.

12. El aprovechamiento de posibles opcionalidades, subjetividades y vacíos en los BT son una herramienta de negocio legítima.

13. El aprovechamiento de posibles opcionalidades, subjetividades y vacíos en los BT constituyen un problema serio y de difícil solución. 


\section{1-Nada 2-Algo 3-Mucho}

14. Para cada uno de los siguientes ítems, señale cuál cree usted que es el grado de manipulación contable al cual se pueden someter: Provisiones para la deducción de activos, Provisiones de pasivos, Gastos de investigación y desarrollo, Derechos de Marcas y/o concesiones, Operaciones de leasing financiero, Menor y/o mayor valor de inversiones, Capitalización de intereses (relacionados con activos fijos), Depreciación de activos fijos, Valoración de inventarios, Reconocimiento de gastos, Reconocimiento de ingresos, Conversión en moneda extranjera, Transacciones entre empresas relacionadas.

15. Ante una empresa que ha manipulado sus estados financieros, indique cual cree usted que es el grado de culpabilidad de los siguientes agentes vinculados a la contabilidad: Auditores externos de estados financieros, Gobierno y administración de la empresa (Gerencias y Directorio), Reguladores entorno a la contabilidad (Colegio de Contadores y/o SVS).

16. Ante el supuesto que en Chile "sí hay manipulación de estados financieros", indique para cada uno de los tipos de empresas detallados, en qué grado estaría ocurriendo: Empresas pequeñas, Empresas medianas y grandes que SI cotizan en bolsa, Empresas medianas y grandes que NO cotizan en bolsa, Entidades públicas.

17. Ante el supuesto que en Chile "sí hay manipulación de estados financieros", indique para cada uno de los sectores económicos detallados, en qué grado estaría ocurriendo: Minero, Forestal, Financiero, Telecomunicaciones, Petróleo, combustible, Obras y construcciones, Manufactura, Retail (supermercados, grandes tiendas, etc.).

18. Considerando que el Colegio de Contadores se encuentra en etapa de convergencia hacia las Normas Internacionales de Contabilidad (NIC/NIIF), usted observa que con la futura aplicación de las NIC/NIIF: Se limitan las posibles opcionalidades de los BT, Se limitan posibles subjetividades de los BT, Se solucionan los vacíos normativos de los BT

$$
\text { (A) }
$$

